\title{
用触針下分布电阻的光电导相位法測量 半导体中非平衡載流子寿命*
}

洪坚

到目前为止, 牛导体中非本衡载流子寿 命测试方法近三十种 $[1,2]$. 这些方法一般可 分为定态法 ${ }^{[3]}$ (郎间接测量) 和非定态法 (郎 直接测量 $)^{[3-11]}$ 两大类. 本交所讨论的测试 法是属于非定态法测量这一类.

当半导体表面受到正弦调制光照时, 如 果样品满足半无限大假定, 那么牛导体內部 非本衡载流子浓度 $(n)$ 应满足连续性方程:

$$
\begin{aligned}
\frac{\partial n}{\partial t}= & D \frac{\partial^{2} n}{\partial x^{2}}-\frac{n}{\tau}+ \\
& +I_{0} \beta k e^{-k x}\left(1+e^{j \omega t}\right)
\end{aligned}
$$

式中 $t$ 表示时间， $x$ 表示沿光照方向坐标， $\tau$ 是样品寿命, $D$ 是双极扩散常数, $I_{0}$ 是单 位时间、单位光照面上平均入射的光子数, $\beta$ 是量子产额, $k$ 是激发光的吸收系数, $\omega$ 是调制的频率.

令方程 (1)的解具有下列形式:

$$
n(x, t)=n_{0}(x)+n_{1}(x) e^{j \omega t}
$$

式 (2) 中 $n_{0}(x)$ 表示与时间无关的电子浓度 (假定空穴浓度亦可), 我们所需要的是与时 间有关的解:

$$
\frac{d^{2} n_{1}(x)}{d x^{2}}-\frac{1}{L^{*^{2}}} n_{1}(x)=-\frac{1}{D} I_{0} \beta k e^{-k x}
$$

边界条件:

$$
\left.\begin{array}{ll}
\frac{x}{L} \rightarrow \infty, & n_{1}(x) \rightarrow 0 \\
x \rightarrow 0, & \left.D \frac{d n_{1}(x)}{d x}\right|_{x=0}=S \cdot n_{1}(0)
\end{array}\right\}
$$

式中 $S$ 是光照面的表面复合速度, $L^{*}=$ $\frac{L}{\sqrt{1+j \omega \tau}}(L$ 是非平衡载流子的扩散长度 $)$, 不难求得方程 (3)的解:

$$
\begin{aligned}
n_{1}(x)= & \frac{1}{D}\left(\frac{L^{*^{2}}}{1-k^{2} L^{*^{2}}}\right) I_{0} \beta k\left\{e^{-k x}-\right. \\
& \left.-\frac{S+k D}{S+\frac{D}{L^{*}}} e^{-\frac{x}{L^{*}}}\right\}
\end{aligned}
$$

作坐标变換, 采用球面极坐标括选触点 作新坐标原点, $x=r \cos \theta$.

为便于观察样品的光电导, 在样品上加 一恆定很小的直流电流 (串联上一个比样品 体电阻大得多的电阻 $R_{s}$, 如 $R_{s} \geqslant 10 R_{0}$ 師可 满足恆流条件), 假定不注入, 等位面仍是球 面, 以及恆定电阻变化与交变电阻变化可以 线性迭加, 光照引起触针下分布电阻变化:

$$
\begin{aligned}
\Delta R=- & \frac{q \mu_{p}\left(1+b_{0}\right)}{2 \pi \sigma_{0}^{2}} \int_{r_{0}}^{\infty} d r \int_{0}^{\frac{\pi}{2}} \frac{n_{1}(r, \theta)}{r^{2}} \times \\
& \times \sin \theta d \theta
\end{aligned}
$$


式中 $q$ 是电子电荷, $r_{0}$ 是触针半径, $\sigma_{0}$ 是无 光照时样品的电导率, $\mu_{p}$ 是空穴迁移率, $b_{0}=\frac{\mu_{n}}{\mu_{p}}$ 是电子、空穴迁移率比值. 以上运 算作了无陷阱假定 (一般陷阱可加底光照消 除), $r_{0} \approx 10^{-3} \mathrm{~cm}$ 是一个很小的量, 在 $\Delta R$ 结果表达式的计算中可略去 $\left(\frac{r_{0}}{L}\right)^{2}$ 以上各 项，利用分部积分计算 (6)式，下面给出四 种不同测试条件的计算结果:

1. 小表面复合 $\left(s \ll \frac{D}{L}, s \ll k D\right)$ 和表面 光激发 $\left(k r_{0} \geqslant 6\right)$ 情形:

这相当于表面腐蚀和用水滤波情形, 光 电导和激发光讯号之间位相差 $\varphi$ 满足以下关 系:

$$
\tan \varphi=-\frac{\omega \tau}{1+\sqrt{1+\omega^{2} \tau^{2}}}
$$

2. 小表面复合 $\left(s \ll \frac{D}{L}, s \ll k D\right)$ 和贯穿 光激发 $(k L \ll 1)$ 情形:

$$
\tan \varphi=-\omega \tau
$$

3. 大表面复合 $\left(s \gg \frac{D}{L}, S \gg k D\right)$ 和长波 光激发 $\left(k r_{0}<1\right)$ 情形:

这相当于粗磨表面和加一定厚度同样品 材料的滤波片, 计算时, 必须把透过滤波片 的不同波长的激发光对光电 导贡献迭加起 来.

$$
\begin{aligned}
\Delta R & =-\frac{2 \pi A_{0} \epsilon_{T}}{4 C^{2}} \int_{0}^{\nu_{0}} f(\nu)[B-j C] d \nu \\
\tan \varphi & =-\frac{\int_{0}^{\nu_{0}} f(\nu) C d \nu}{\int_{0}^{\nu_{0}} f(\nu) B d \nu}= \\
& =-\eta\left(L_{1}, \frac{r_{0}}{L_{1}}, \omega \tau, d_{0}\right) \cdot \omega \tau
\end{aligned}
$$

其中

$$
\begin{aligned}
& a^{2}=\frac{1}{2}\left[\sqrt{1+\omega^{2} \tau^{2}}+1\right] \\
& b^{2}=\frac{1}{2}\left[\sqrt{1+\omega^{2} \tau^{2}}-1\right] \\
& A_{0}=\frac{q \mu_{p}\left(1+b_{0}\right) \beta}{2 \pi \sigma_{0}^{2} D} \\
& -E i(-x) \equiv \int_{x}^{\infty} \frac{1}{t} e^{-t} d t \\
& B=\left(1-k^{2} L^{2}\right) X+2 a b Y \\
& C=2 a b X-\left(1-k^{2} L^{2}\right) Y \\
& X=V-\frac{4 r_{0}}{3 L}\left(1+\frac{3 b^{2}}{2}\right)- \\
& -W-2 a E_{i}\left(-\frac{r_{0}}{L}\right) \\
& Y=U-\frac{2 a b}{3}\left(\frac{r_{0}}{L}\right)-2 b E i\left(-\frac{r_{0}}{L}\right) \\
& V=\frac{1}{a}\left[3 a^{2}+b^{2}\left(1-\frac{b^{2}}{6 a^{2}}+\frac{b^{4}}{15 a^{4}}\right)\right] \\
& U=b\left[1-\frac{b^{2}}{3 a^{2}}+\frac{b^{4}}{10 a^{4}}-\frac{b^{6}}{3 a^{6}}\right] \\
& W=3 k L-\frac{4}{3} k^{2} L r_{0}+\frac{1}{3} k^{3} L r_{0}^{2}- \\
& -2 k L E i\left(-k r_{0}\right) \\
& f(\nu)=\frac{\nu^{2}}{e^{\frac{h \nu}{k_{0} \tau}}-1} \cdot \frac{k L}{\left(1-k^{2} L^{2}\right)^{2}+4 a^{2} b^{2}} \cdot \\
& \text { - } D_{0}(v)
\end{aligned}
$$

式中 $\nu$ 是激发光频率, $D_{0}(v)=e^{-k d_{0}}$ 是滤 波片在不同频率下的透过率 ${ }^{[12]}, d_{0}$ 是滤波片 厚度, $C$ 是光速, $h$ 是普朗克常数, $k_{0}$ 是玻 尔茲曼常数, $e_{T}$ 是光谱光源与黑体辐射光 谱间的一个比例常数 (在我们所研究的波长 范围內可以假设 $\epsilon_{T}$ 与 $\nu$ 无关).

4. 大表面复合 $\left(S \gg \frac{D}{L}, S \gg k D\right)$ 和贯穿 光激发 $(k L \gg 1)$ 情形:

$$
\tan \varphi=-\frac{1-\eta_{0}}{1+\eta_{0} \omega^{2} \tau^{2}} \omega \tau
$$




$$
\eta_{0}=\frac{1}{2} \cdot \frac{1-\frac{b^{2}}{3 a^{2}}+\frac{b^{4}}{10 a^{4}}-\frac{b^{6}}{3 a^{6}}-\frac{2 a r_{0}}{3 L}-2 E i\left(-\frac{r_{0}}{L}\right)}{3 a^{2}+b^{2}\left(1-\frac{b^{2}}{6 a^{2}}+\frac{b^{4}}{15 a^{4}}\right)-\frac{4 a r_{0}}{L}\left(1+\frac{3 b^{2}}{2}\right)-2 a^{2} E i\left(-\frac{r_{0}}{L}\right)}
$$

实际上, 我们朵用 $S \rightarrow \infty$ (大表面复合) 和 $k r_{0}<1$ (长波激发) 测量条件, 我们对硅 材料进行了数値计算, 计算结果列于 表 1 、 $2 、 3$ 和图 1、2 中.

表 $1 \frac{r_{0}}{L}=0.05, d_{0}=0.5 \mathrm{~cm}$

\begin{tabular}{c|c|c|c|c|c|c}
\hline$\eta$ & $\omega \tau$ & & & & & \\
$L(\mathrm{~cm})$ & & 0.5 & 1 & 2 & 3 & 5 \\
\hline $1 \times 10^{-2}$ & 0.613 & 0.586 & 0.525 & 0.412 & - & - \\
\hline $3.376 \times 10^{-2}$ & 0.593 & 0.562 & 0.506 & 0.394 & 0.315 & 0.226 \\
\hline $7 \times 10^{-2}$ & 0.566 & 0.537 & 0.483 & 0.377 & 0.301 & 0.218 \\
\hline $1.068 \times 10^{-1}$ & 0.539 & 0.512 & 0.460 & 0.368 & 0.291 & 0.210 \\
\hline $1.38 \times 10^{-1}$ & 0.523 & 0.499 & 0.454 & 0.355 & 0.290 & 0.208 \\
\hline
\end{tabular}

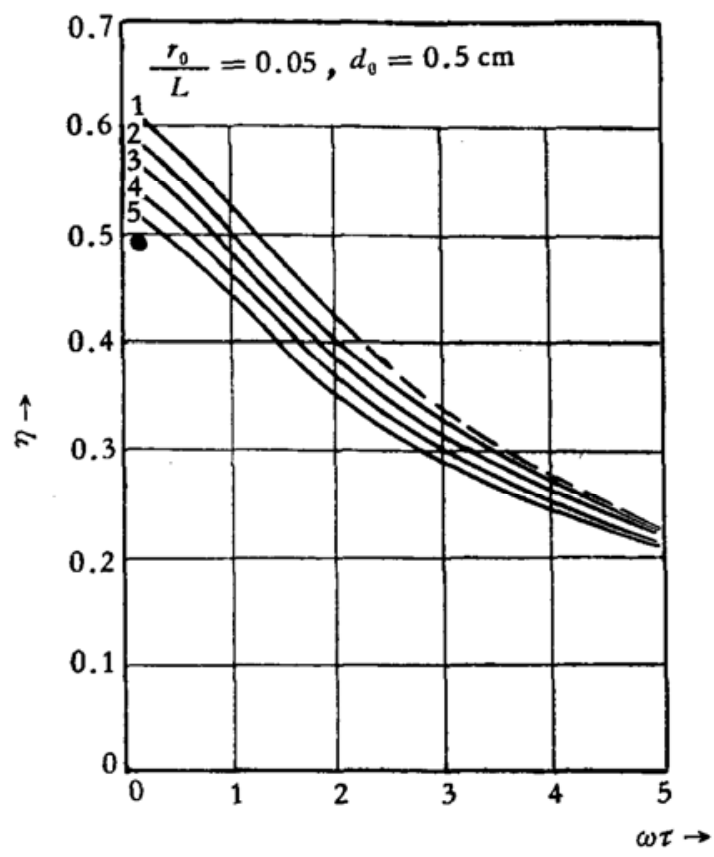

图 $1 S \rightarrow \infty, k r_{0}<1$ 情形 $\eta-\omega \tau$ 曲线 曲线 $1 . L=1 \times 10^{-2} \mathrm{~cm}$ 曲线 2. $L=3.376 \times 10^{-2} \mathrm{~cm}$ 曲线 3. $L=7 \times 10^{-2} \mathrm{~cm}$ 曲线 4. $L=1.068 \times 10^{-1} \mathrm{~cm}$ 曲线 5. $L=1.38 \times 10^{-1} \mathrm{~cm}$
表 $2 \frac{r_{0}}{L}=0.01, d_{0}=0.5 \mathrm{~cm}$

\begin{tabular}{|c|c|c|c|c|c|c|}
\hline & 0.1 & 0.5 & 1 & 2 & 3 & 5 \\
\hline $3.376 \times 10^{-2}$ & 0.554 & 0.528 & 0.471 & 0.372 & 0.288 & 0.206 \\
\hline $1.068 \times 10^{-1}$ & 0.502 & 0.487 & 0.432 & 0.336 & 0.262 & 0.191 \\
\hline
\end{tabular}

表 $3 \frac{r_{0}}{L}=0.05, d_{0}=0.1 \mathrm{~cm}$

\begin{tabular}{|c|c|c|c|c|c|c|}
\hline & 0.1 & 0.5 & 1 & 2 & 3 & 5 \\
\hline $3.376 \times 10^{-2}$ & 0.498 & 0.488 & 0.442 & 0.348 & 0.282 & 0.202 \\
\hline
\end{tabular}

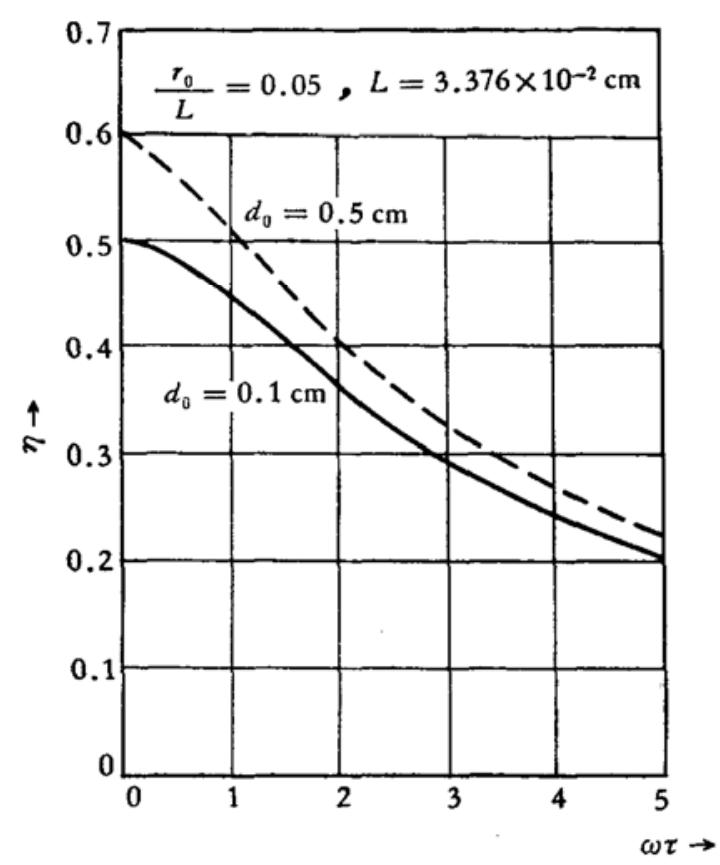

图 $2 s \rightarrow \infty, k r_{0}<1$ 情形下, 不同厚 度硅滤片的 $\eta \sim \omega \tau$ 曲线

实验方框图如图 3 所示. 我们采用转盘 调制光, 光源是用 400 瓦铇丝灯, 转盘孔眼 为正弦形, 只要转盘直径足够大, 那么照在 样品上的光強正比于透光面积. 我们曾用光 


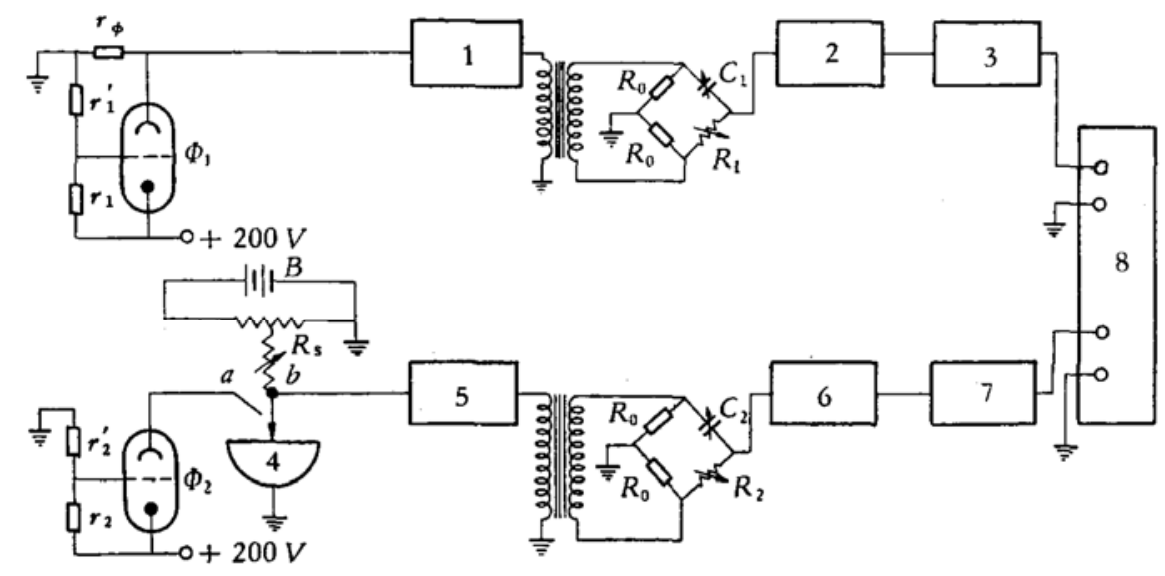

图 3 相位测试装置图

\section{1. 阴极输出; 2. 阴极输出; 3. 选频放大; 4.样品; 5. 阴极输出; 6. 阴极输出; \\ 7.选频放大; 8 . 相表}

电管接收讯号并直接显示在示波屏上，证实 激发光讯号确是正弦形.

样品表面用 304 号金钢砂研磨，底部涂 擦金一鎵合金做成欧姆接触，硅滤片选用 $5 \mathrm{~mm}$ 厚（低阻短寿命样品可改用 $1 \mathrm{~mm}$ 厚 硅片).

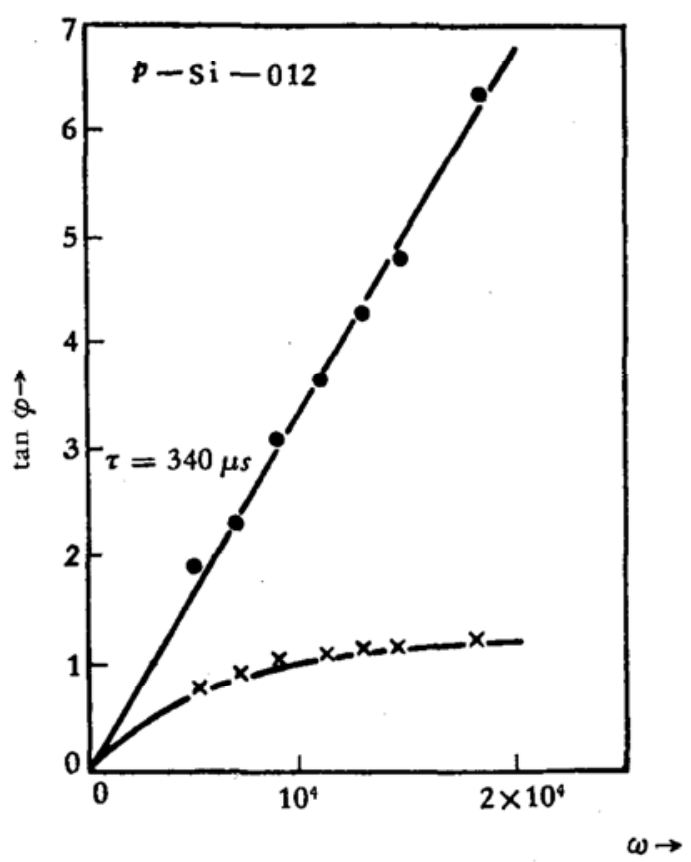

图 $4 S \rightarrow \infty, k r_{0}<1$ 情形 $\tan \varphi \sim \omega$ 曲线 ( $5 \mathrm{~mm}$ 厚硅滤片 P-Si-012, $\rho=5,600 \Omega-\mathrm{cm}$ ) $\times$ 实验点 - 修正后
图 4 是 P-Si 矩形样品 $(8 \times 6 \times 20 \mathrm{~mm})$ 测试结果, 电阻率为 $5,600 \Omega-\mathrm{cm}$. 严格说 来, 这块样品还不完全满足半无限大样品假 定, 测试结果 $\tau=340 \mu \mathrm{s}$ (光电导测得 $\tau=$ $300 \mu s$ ). 图 5 也是 P-Si 矩形样品 (尺寸比 P-Si-012 更窄更薄）测试曲线图, 测得 $\tau=$

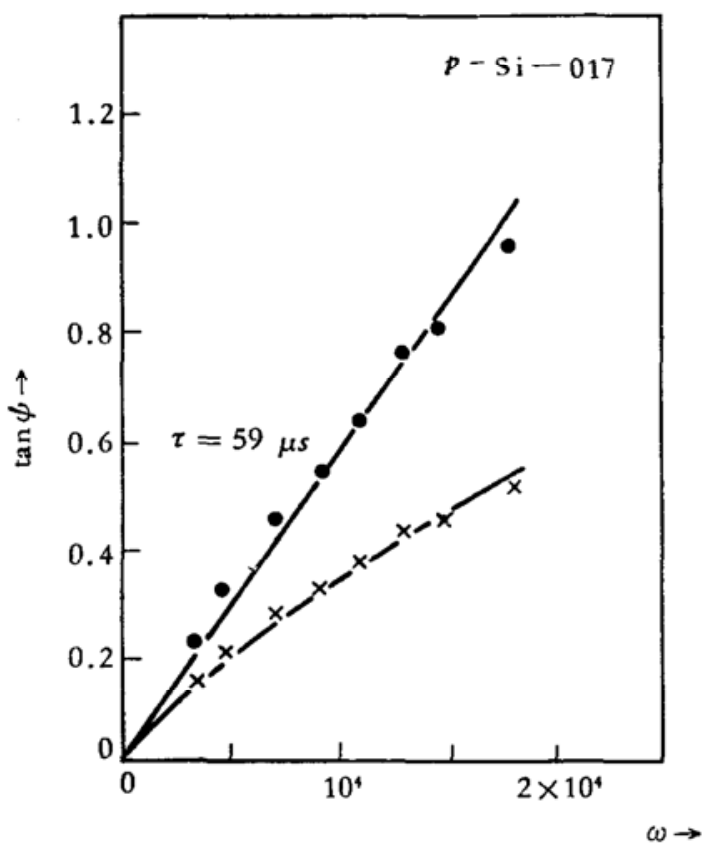

图 $5 s \rightarrow \infty, k r_{0}<1$ 情形 $\tan \varphi \sim \omega$ 曲线 ( $5 \mathrm{~mm}$ 厚硅淧片 P-Si-017, $\rho=4,000 \Omega-\mathrm{cm}$ ) $\times$ 实验点

- 修正后 
$57 \mu s$ (光电导测得 $\tau=45 \mu s$ ). 同时, 我们 也对低阻 $n$ 型和 $p$ 型硅以及 $n-\mathrm{Ge}$ 样品进行 测量 (对锗样品采用腐蚀表面和 $3 \mathrm{~cm}$ 厚水滤 波), 测得结果基本上同光电导法、双脉冲 法测量结果相一致.

$$
\text { 由于 } r_{0} \text { 很小，一般 } 0.01 \leqslant \frac{r_{0}}{L} \leqslant 0.05 \text {, }
$$

我们作了具体计算, 按 $\frac{r_{0}}{L}=0.05$ 修正所引 进误差可予以忽略. 为了实际测量方便, 把

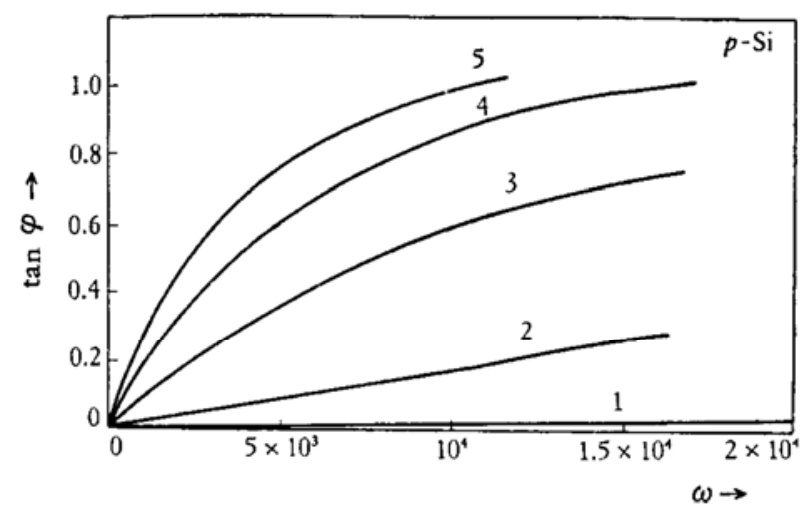

图 $6 \mathrm{P}-\mathrm{Si} \tan \varphi \sim \omega$ 理论曲线 (未修正) 曲线 1. $\tau=264 \mu \mathrm{s}$; 曲线 2. $\tau=30 \mu \mathrm{s}$; 曲线 3. $\tau=129 \mu \mathrm{s}$; 曲线 $4 . \tau=300 \mu \mathrm{s}$; 曲线 5 . $\tau=500 \mu \mathrm{s}$

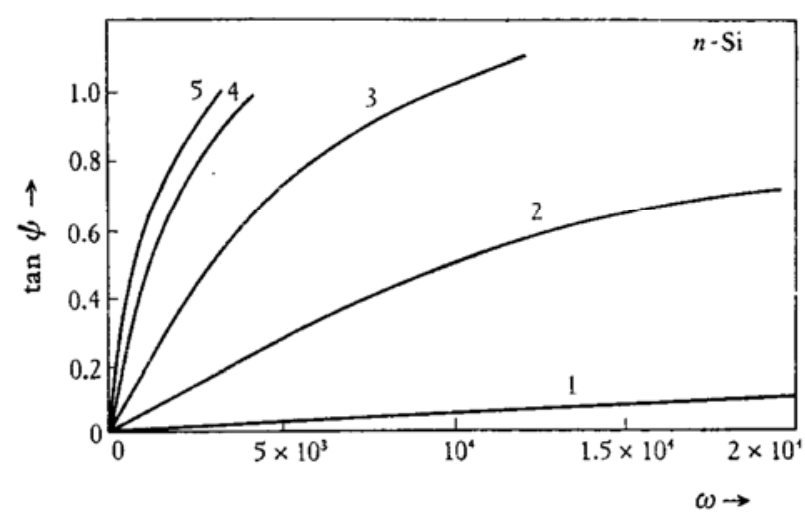

图 $7 \mathrm{n}-\mathrm{Si} \tan \varphi \sim \omega$ 理论曲线 (未修正) 曲线 1. $\tau=7.7 \mu \mathrm{s}$; 曲线 2. $\tau=88 \mu \mathrm{s}$; 曲 线 3. $\tau=376 \mu \mathrm{s}$; 曲线 4. $\tau=880 \mu \mathrm{s}$; 曲 线 5. $\tau=1470 \mu \mathrm{s}$
图 1 中五条修正曲线变換为 $\tan \varphi \sim \omega$ 曲线, 㪶且分別按 $p$ 型硅和 $n$ 型硅画出. 结果如图 $6 、 7$ 所示.

通过详细分析表明: 本法所测得寿命乃 是触点附近局部寿命（主要贡献区大约离触 点五倍扩散长度以內)。本法除了具备相位 移法高灵敏度优点外还具有下列优点: 1 . 可 以在锭状晶体上测量; 2 . 表面处理十分简 单；3. 在样品上不需制作固定电极； 4. 测量 仪器简单, 操作方便; 5. 有一定准确度.

这个方法可以检验不均匀材料, 可供科. 学㸴究机关作研究用, 更适于工厂检验单晶 材料用.

本工作是在王守武副所长亲自指导下进行的. 承王启明、周汝生、高季鱗、刘延卿、郁元桓、曹 秉发、庄蔚华等同志大力协助和支持，使工作得以 顺利完成, 作者深表感谢.

\section{参考 文 献}

[1] 王守武, 物理学报, 19, 176 (1963).

[2] Копыловский Б. Д., Заводская лаборатория, 30, 289 (1964).

[3] Garreta, O. \& Grosvalet, J., Progress in Semicon, Ductors, 1, 167 (1956).

[4] Богданов С. В., Копыловский Б. Д., Ф. Т. Т., 3, 926 (1961).

[5] Копыловский Б. Д., Богданов С. В., Ф. Т. Т., 4, 2867 (1962).

[6] Suryan, G., Susila, G. Miss, J. Scient. \& Instr. Res., A 21, 235 (1962).

[7] Schultz, B. H., Philips. Res. Rep., 10, 337 (1955).

[8] Van der Pauw, L. J., Philips. Res. Rep., 12, 364 (1957).

[9] Ridout, M. S., Halbleiter und Phosphore, 408, 1956.

[10] Choo, S. C., Heasell, E. L., Rev. Sci. Instr., 33, 1331 (1962).

[11] Wertheim, G. K., Phys. Rev., 109, 1086 (1958).

[12] Dash, W. C., Newman, R., Phys. Rev., 99, 1151 (1955). 